\title{
$\gamma$-Secretase Inhibition Reduces Spine Density In Vivo via an Amyloid Precursor Protein-Dependent Pathway
}

\author{
Tobias Bittner, ${ }^{1}$ Martin Fuhrmann, ${ }^{1}$ Steffen Burgold, ${ }^{1}$ Christian K. E. Jung, ${ }^{1}$ Christiane Volbracht, ${ }^{2}$ Harald Steiner, ${ }^{3}$ \\ Gerda Mitteregger, ${ }^{1}$ Hans A. Kretzschmar, ${ }^{1}$ Christian Haass, ${ }^{3}$ and Jochen Herms ${ }^{1}$ \\ ${ }^{1}$ Center of Neuropathology and Prion Research, Ludwig-Maximilians-Universität, 81377 Munich, Germany, ${ }^{2}$ Department of Molecular Neurobiology, \\ H. Lundbeck, 2500 Valby, Denmark, and ${ }^{3}$ German Centre for Neurodegenerative Diseases (DZNE)-Munich and Department of Biochemistry, Adolf- \\ Butenandt-Institute, Ludwig-Maximilians-Universität, 80336 Munich, Germany
}

\begin{abstract}
Alzheimer's disease $(\mathrm{AD})$ represents the most common age-related neurodegenerative disorder. It is characterized by the invariant accumulation of the $\beta$-amyloid peptide $(\mathrm{A} \beta)$, which mediates synapse loss and cognitive impairment in AD. Current therapeutic approaches concentrate on reducing $\mathrm{A} \beta$ levels and amyloid plaque load via modifying or inhibiting the generation of $\mathrm{A} \beta$. Based on in vivo two-photon imaging, we present evidence that side effects on the level of dendritic spines may counteract the beneficial potential of these approaches. Two potent $\gamma$-secretase inhibitors (GSIs), DAPT ( $N$-[ $N$-(3,5-difluorophenacetyl-L-alanyl)]-S-phenylglycine $t$-butyl ester) and LY450139 (hydroxylvaleryl monobenzocaprolactam), were found to reduce the density of dendritic spines in wild-type mice. In mice deficient for the amyloid precursor protein (APP), both GSIs had no effect on dendritic spine density, demonstrating that $\gamma$-secretase inhibition decreases dendritic spine density via APP. Independent of the effects of $\gamma$-secretase inhibition, we observed a twofold higher density of dendritic spines in the cerebral cortex of adult APP-deficient mice. This observation further supports the notion that APP is involved in the modulation of dendritic spine density—-shown here for the first time in vivo.
\end{abstract}

\section{Introduction}

Alzheimer's disease (AD) is the most common neurodegenerative disorder in the elderly. Pathognomonic features include the accumulation of amyloid $\beta$-peptide $(\mathrm{A} \beta)$, neurofibrillary tangles, neuronal death, and synapse loss (Terry et al., 1991; Selkoe, 2002). The amyloid precursor protein (APP) is of central importance in $\mathrm{AD}$ pathogenesis because it represents the precursor of $\mathrm{A} \beta$ as the prime causative agent (Selkoe, 1994). APP is first cleaved by the $\beta$-site APP-cleaving enzyme 1 (BACE1), resulting in a secreted ectodomain of APP and the 99 aa C-terminal fragment $\beta(\mathrm{CTF} \beta)$. The latter is subsequently processed by $\gamma$-secretase to generate the APP intracellular domain and A $\beta$, which is secreted into biological fluids (Haass, 2004). A major therapeutic approach, which is currently under clinical testing, is the pharmacological inhibition of $\gamma$-secretase (Selkoe and Wolfe, 2007). To assess the impact of $\gamma$-secretase inhibitors (GSIs) on individual synapses, we performed transcranial two-photon imaging in the cortex of living mice. Long-term in vivo imaging provides a powerful tool to explore structural plasticity of single synapses over extended time periods in the cerebral cortex of

Received May 15, 2009; revised July 8, 2009; accepted July 14, 2009.

This work was supported by grants from the Deutsche Forschungsgemeinschaft (SFB 596), the Bundesministerium für Bildung und Forschung (01GZ0713;16IN0675 13N9268), and the European Union (FP7-223276). This work was further supported by the Center of Integrated Protein Science Munich. We thank A. Giese (University of Munich) and N. Vassallo (University of Malta) for comments on this manuscript. We also thank B. Lindquist (Stony Brook University) for providing the 3DMA software.

Correspondence should be addressed to Dr. Jochen Herms, Center of Neuropathology and Prion Research, Ludwig-Maximilians-Universität, Feodor-Lynen-Strasse 23, 81377 Munich, Germany. E-mail: jochen.herms@ med.uni-muenchen.de.

DOI:10.1523/JNEUROSCI.2288-09.2009

Copyright $\odot 2009$ Society for Neuroscience $\quad$ 0270-6474/09/2910405-05\$15.00/0 living mice (Grutzendler et al., 2002; Trachtenberg et al., 2002; Tsai et al., 2004; Spires et al., 2005; Zuo et al., 2005; Holtmaat et al., 2006; Fuhrmann et al., 2007). This method thus enables direct monitoring of the effect of drugs on structural plasticity of individual spines during and after drug administration. Specifically, dendritic spines in apical dendrites of layer 3 and layer 5 neurons of the somatosensory cortex were analyzed in wild-type (WT) mice to assess the impact of the $\gamma$-secretase inhibitors DAPT $(\mathrm{N}-[\mathrm{N}-(3,5-$ difluorophenacetyl-L-alanyl)]-S-phenylglycine $t$-butyl ester) and LY450139 dihydrate (hydroxylvaleryl monobenzocaprolactam), which is in clinical trials (Fleisher et al., 2008), on structural plasticity of dendritic spines.

\section{Materials and Methods}

Mice. $A P P^{-/-}$mice (Li et al., 1996) were crossed with heterozygous mice of the YFP-H line (Feng et al., 2000) (The Jackson Laboratory). The $A P P^{-/-}$offspring was backcrossed with $A P P^{+/-}$mice to yield littermate $A P P^{-1-}$ and $A P P^{+/-}$mice. As controls we used mice of the same background, but that were $A P P^{+/+}$. All mice were heterozygous for YFP and of mixed gender, and were analyzed at 4-6 months of age. Mice were group housed under pathogen-free conditions until surgery, after which they were singly housed. All procedures were in accordance with an animal protocol approved by the University of Munich and the government of upper Bavaria.

Cranial window surgery. A cranial window over the right cortical hemisphere was surgically implanted as previously described (Fuhrmann et al., 2007). The mice were anesthetized with an intraperitoneal injection of ketamine/xylazine $(0.13 / 0.01 \mathrm{mg} / \mathrm{g}$ body weight). Additionally, dexamethasone $(0.02 \mathrm{ml}$ at $4 \mathrm{mg} / \mathrm{ml})$ was intraperitoneally administered immediately before surgery (Holtmaat et al., 2005). A circular piece of the skull over the somatosensory cortex (4 $\mathrm{mm}$ in diameter) was removed using a dental drill (Schick-Technikmaster C1; Pluraden). Immediately, 

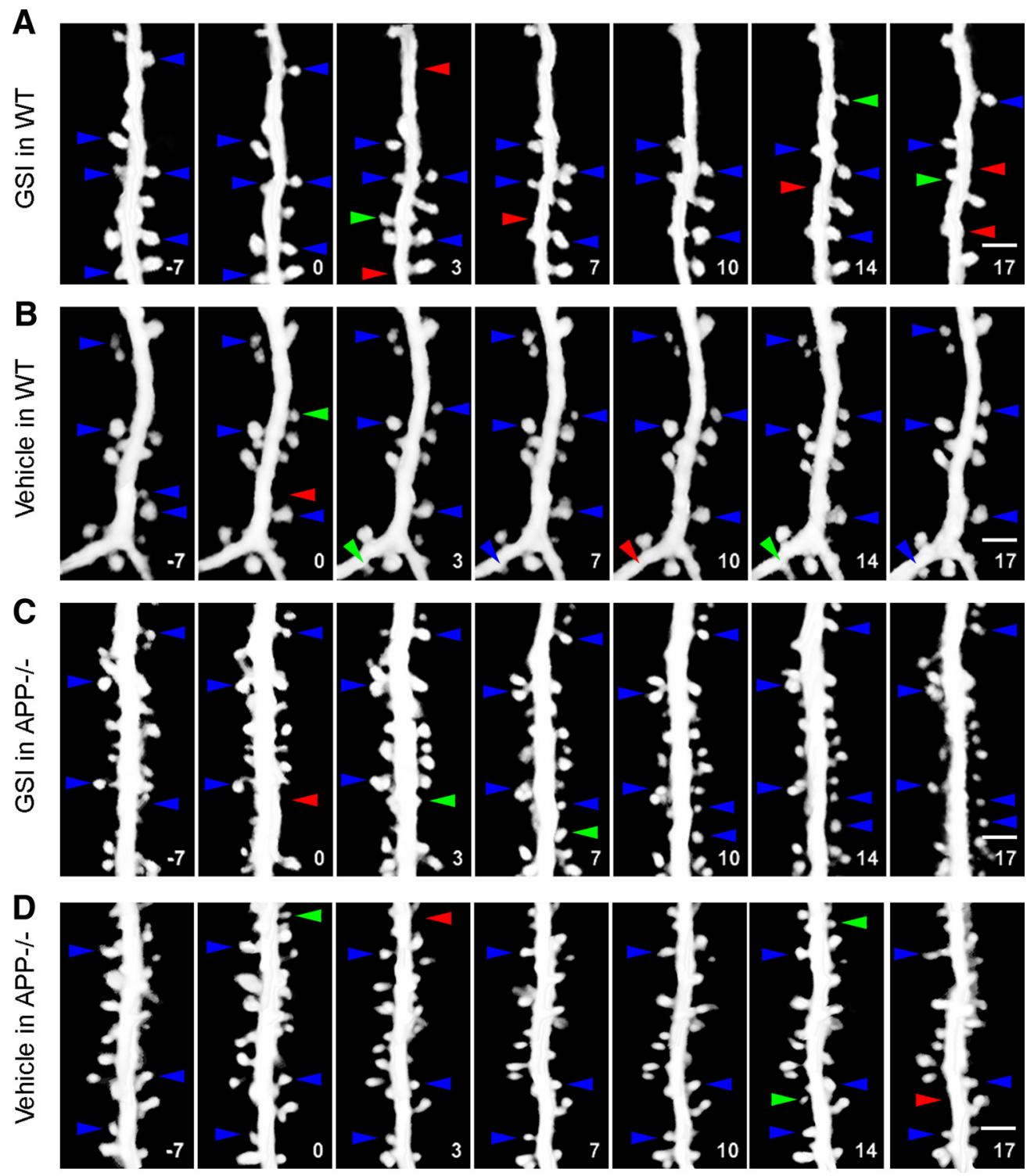

Figure 1. The fate of individual dendritic spines before and after GSI treatment in WT and $A P P$ KO mice $\left(A P P^{-I-}\right) \cdot A-D, 0$ ver a time period of 25 d, representative two-photon in vivo images were taken of the same dendritic element in 4- to 6-month-old mice. Numbers indicate time points in days relative to day 0 when the treatment (LY450139; $30 \mathrm{mg} / \mathrm{kg}$ ) started for $4 \mathrm{~d}$. Blue arrows exemplarily mark spines that were stable at that exact time point; green arrows indicate gained spines, whereas red arrows represent lost spines at that time point. Scale bars, $3 \mu \mathrm{m}$. $A, G S I$-treated WT mice; $\boldsymbol{B}$, vehicle-treated WT mice; $\boldsymbol{C}$, GSI-treated APP KO mice; and $\boldsymbol{D}$, vehicle-treated APP KO mice.

a round coverslip (5 $\mathrm{mm}$ diameter) was glued to the skull using dental acrylic (Cyano-Veneer fast; Schein) to close the craniotomy. A small metal bar, containing a hole for a screw, was glued next to the coverslip to allow repositioning of the mouse during subsequent imaging sessions. After surgery, mice received a subcutaneous analgesic dose of carprophen (Rimadyl; Pfizer) for $3 \mathrm{~d}(5 \mathrm{mg} / \mathrm{kg}$ ). Imaging began after a $21 \mathrm{~d}$ rest period after surgery for the window to become stable. The mice were killed at the end of the experiment by cervical dislocation.

$\gamma$-Secretase inhibitor administration. DAPT (Millipore Bioscience Research Reagents, Merck) was formulated in 5\% (v/v) ethanol and corn oil and subcutaneously administered in $100 \mathrm{mg} / \mathrm{kg}$ (Dovey et al., 2001) daily for $4 \mathrm{~d}$. LY450139 (Gitter et al., 2004) was synthesized following the schemes provided by Lilly and formulated in 10\% Cremophor EL solution (Sigma-Aldrich), and administered subcutaneously in $30 \mathrm{mg} / \mathrm{kg}$ (Ness et al., 2004) daily for $4 \mathrm{~d}$.

Two-photon in vivo imaging. In vivo two-photon imaging was performed in 3- to 4-d intervals after a $21 \mathrm{~d}$ rest period after cranial window surgery (Fuhrmann et al., 2007). An Axioskop 2Fs mot LSM510Meta/ NLO setup (Zeiss), equipped with a MaiTai laser (Spectra Physics), was used. The MaiTai Ti:Saphir laser had a bandwidth of 710-920 nm and was pumped by a $10 \mathrm{~W}$ solid-state laser (Millenia X; Spectra Physics). Yellow fluorescent protein (YFP) was excited at $880 \mathrm{~nm}$. For in vivo imaging, mice were anesthetized by an intraperitoneal injection of ketamine/xylazine, $0.01 / 0.0075 \mathrm{mg} / \mathrm{kg}$ body weight). Imaging sessions lasted for no longer than $60 \mathrm{~min}$. Image acquisition was performed with the Zeiss LSM510 Rel3.2 software. For overview images, $z$-stacks of 150 $\mu \mathrm{m}$ with $2 \mu \mathrm{m} z$-resolution and $1024 \times 1024$ pixels per image frame $(0.22 \mu \mathrm{m} /$ pixel $)$ were taken with a Zeiss $40 \times$ water-immersion objective (0.8 numerical aperture). For higher-resolution images to count dendritic spines, the same objective was used. Image stacks of various sizes (10-50 $\mu \mathrm{m}$ depth) with $1 \mu \mathrm{m} z$-resolution and $512 \times 512$ pixels per image frame $(0.11 \mu \mathrm{m} /$ pixel $)$ were generated. Care was taken to ensure similar fluorescence levels in space and time. Apical dendritic tufts in layer 1-2 of the somatosensory cortex were imaged. Repositioning of the same dendritic element over time was achieved by orienting to the vascular pattern and unique branch points of apical dendrites. Five to 10 positions per mouse, containing several dendritic elements and neurons up to $200 \mu \mathrm{m}$ below the pial surface, were imaged over the indicated time periods.

Image processing and data analysis. All images were deconvolved (AutoDeblur, version X2.0.1, Media Cybernetics) and filtered with an 
A

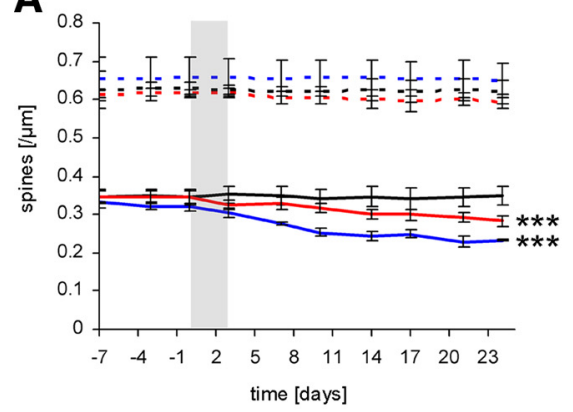

C

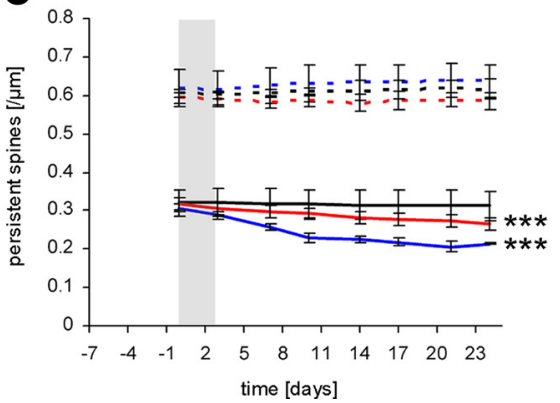

B

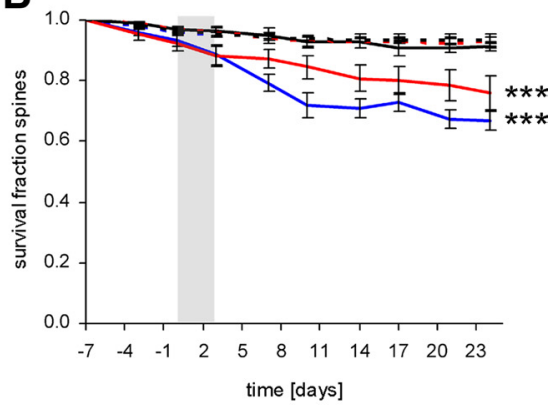

D

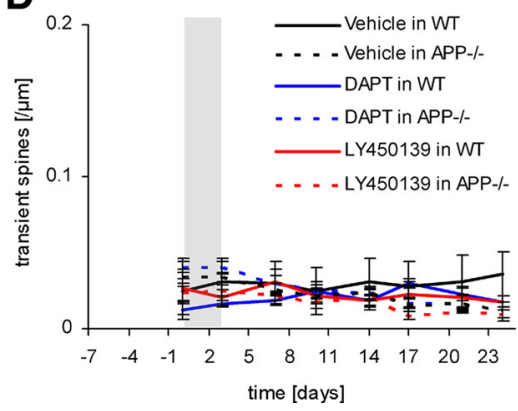

Figure 2. Dendritic spine density decreased after GSI treatment by either DAPT or LY450139, exclusively in WT, but not in APP $K 0$, mice, indicating an APP-dependent mechanism. $\boldsymbol{A}-\boldsymbol{D}$, Time point values represent mean values of individual mice $(n=5 \mathrm{per}$ group). The gray column indicates the $4 \mathrm{~d}$ treatment period (days $0-3$ ). Error bars represent mean \pm SEM. $A$, The average dendritic spine density decreased significantly in WT mice after GSI treatment compared with vehicle-treated WT mice (repeated-measures ANOVA; $p<0.001$ ), whereas in APP KO mice, GSI treatment had no effect on the dendritic spine density. In addition, the baseline dendritic spine density in APP KO mice was elevated by twofold. The severity of the induced dendritic spine loss in WT was stronger by DAPT compared with LY450139. B, The average survival fraction of spines significantly decreased (repeated-measures ANOVA; $p<0.001$ ) exclusively in WT mice after GSI treatment. The decrease in dendritic spine density manifested only in a loss of persistent spines (repeatedmeasures ANOVA; $p<0.001)(\boldsymbol{C}$, whereas in transient spines, no significant difference was detected (D).

A
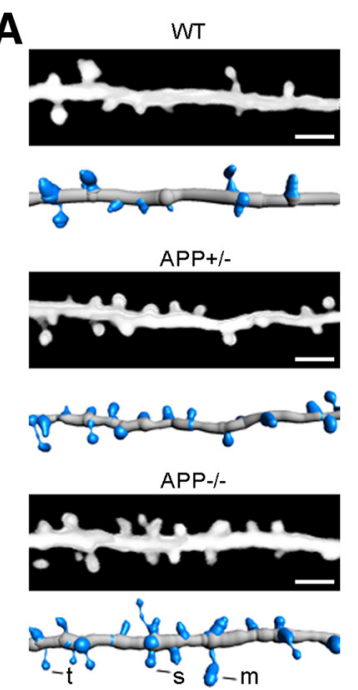

B
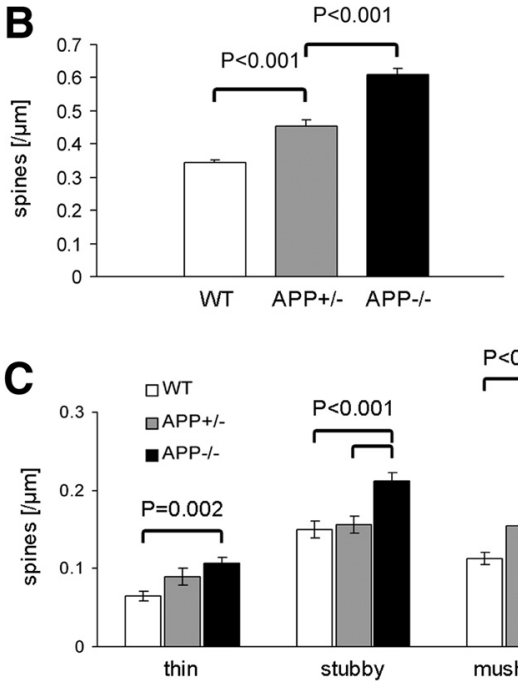

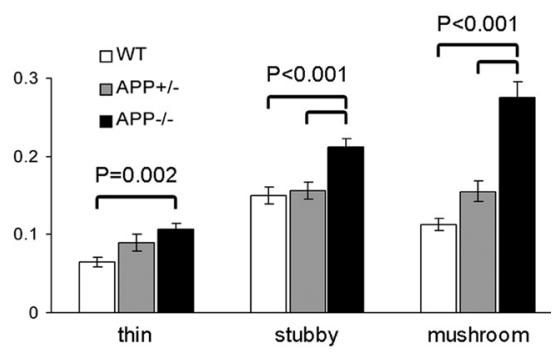

Figure 3. Dendritic spine density and spine shape are dependent on APP expression in a dose-dependent manner. $\boldsymbol{A}$, Representative images of dendritic branches of 4 - to 6-month-old WT, heterozygous APP (APP ${ }^{+1-}$ ), and APP KO (APP $\left.{ }^{-1-}\right)$ mice. Dendritic elements were three-dimensionally reconstructed to classify dendritic spines regarding their shape using a computerbased algorithm. Dendritic spine shapes are exemplarily indicated by $\mathrm{t}$ (thin), $\mathrm{s}$ (stubby), and m (mushroom). Scale bars, $3 \mu \mathrm{m}$. $B$, The dendritic spine density was significantly higher in $A P P^{-1-}$ mice compared with $A P P^{+/-}$littermates and WT mice (one-way ANOVA, $p<0.001$ ). C, The increase in dendritic spine density in $A P P^{-1-}$ mice compared with $A P P^{+1-}$ and WT mice was mainly caused by an increase in stubby- and mushroom-shaped spines (one-way ANOVA). Error bars ( $\boldsymbol{B}, \boldsymbol{C}$ ) indicate SEM.

edge-preserving algorithm, followed by a local contrast change (Imaris 5.0.1, Bitplane). The images were aligned (Stackreg plug-in, NIH ImageJ, version 1.38), and maximum intensity was projected (Zeiss LSM510 3.2Rel software) or three-dimensionally reconstructed (Filament tracer,
Imaris 5.7.2, Bitplane). In some figures, distracting neighboring dendritic elements were removed. Spines were counted in $z$-stacks by manually scrolling through the images of subsequent time points of the same position. All clear protrusions emanating laterally from the dendritic shaft, regardless of shape, were measured. Spines were counted as stable when they were detected at the same place, not $>1 \mu \mathrm{m}$ away from the previous position. Lost spines were counted as lost when they consisted of $<4$ pixels and as gained when they consisted of $>4$ pixels in length protruding from the dendrite. This scoring method has previously been used successfully (Holtmaat et al., 2005; Fuhrmann et al., 2007). Because the $z$-axis resolution of two-photon microscopy is insufficiently low, only protrusions emanating laterally from the dendrite were counted. Dendritic spine analysis was performed in a blind manner by an experimenter without knowledge of the experimental conditions. Spine densities refer to the amount of spines per dendrite length, in micrometers, from which they protrude. Spines lasting for $<8 \mathrm{~d}$ were classified as transient and spines with a lifetime of $8 \mathrm{~d}$ or more as persistent (Holtmaat et al., 2006; Knott et al., 2006). Fraction of spine survival represents the fraction of spines present at a certain time point compared with the first imaging time point. Analysis of spine shape was performed with 3DMA software as previously described (Koh et al., 2002).

Statistics. To test for significant changes over time, repeated-measures ANOVA was performed of individual mice in each group $(n=$ 4-5 mice with a total of 35,420 analyzed dendritic spines in 5-9 dendrites per mouse). To test significance of dendritic spine density and dendritic spine density related to spine shape, one-way ANOVA was performed comparing individual dendritic elements $(n=70$ $\left.A P P^{-1-}, n=36 \mathrm{APP}^{+/-}, n=66 \mathrm{WT}\right)$ with a total of 6224 dendritic spines in $n=29$ mice. All data are presented as mean \pm SEM; asterisks $\left.{ }^{* * *}\right)$ indicate $p<0.001$.

\section{Results}

GSIs are known to reduce $A \beta$ levels in the brain of transgenic Alzheimer mouse models as much as $60 \%$ while significantly increasing APP-CTF levels (Dovey et al., 2001; Ness et al., 2004). In our experiments, GSI treatment led to a significant decrease of dendritic spine density in 4- to 6-month-old WT mice, an effect that was not observed in vehicle-treated mice (Figs. $1 A, B, 2 A$; repeated-measures ANOVA; $p<0.001 ; n=5$ per group). This decline in spine density started directly after GSI treatment and did not recover within 3 weeks after the last application. Furthermore, the GSI-induced dendritic spine loss was associated with a lower survival fraction of dendritic spines compared with vehicle-treated mice (Fig. 2 B). Notably, only the density of persistent spines (lifetime $\geq 8 \mathrm{~d}$ ), which represent the synapse-forming class of spines 
(Holtmaat et al., 2006; Knott et al., 2006) decreased (Fig. 2C; repeated-measures ANOVA; $p<0.001 ; n=5$ per group). The density of transient spines (lifetime $<8 \mathrm{~d}$ ) remained unchanged (Fig. 2D). This indicates that the mechanisms involved in building up new spines are not altered by the GSI treatment.

GSIs block $\gamma$-secretase processing of APP as well as numerous other type I membrane proteins, including Notch (Geling et al., 2002; Kopan and Ilagan, 2004). To investigate whether the GSIinduced dendritic spine loss in WT mice was a consequence of an inhibition of APP processing, mice deficient for APP [APP knock-out $(A P P K O)$ ] were treated with the GSIs in the same way as WT mice. Hereby, we detected that $A P P K O$ mice exhibit a baseline dendritic spine density that is approximately twofold higher compared with WT mice (Fig. $3 A, B$ ). In littermates heterozygous for $\mathrm{APP}\left(A \mathrm{PP}^{+/-}\right)$, dendritic spine density was significantly lower than in APP KO mice but still elevated compared with WT mice (Fig. $3 A, B$; $\mathrm{APP}^{-1-}, 0.59 \pm 0.03 \mu \mathrm{m}^{-1}$ vs $\mathrm{APP}^{+/-}, 0.40 \pm 0.02 \mu \mathrm{m}^{-1}$ vs WT, $0.33 \pm 0.01 \mu \mathrm{m}^{-1}$; one-way ANOVA, $p<0.001 ; n=70$ vs $n=36$ vs $n=60$ dendrites, respectively). This strongly reinforces the evidence that dendritic spine density is modulated in a dose-dependent manner by APP expression. The increased dendritic spine density in APP KO mice was attributable to a higher number of persistent spines (Fig. 2C), whereas no significant difference in transient spine density was observed (Fig. 2D). To support the fact that APP KO mice had more functional synapses compared with WT, we classified the spines according to their shape. APP KO mice displayed an increased level of thin-, stubby-, and mushroom-shaped spines (Fig. 3C). However, especially the amount of stubby- and mushroom-shaped spines was increased, which are mainly persistent spines and therefore represent functional synapses (Holtmaat et al., 2006; Majewska et al., 2006).

After GSI treatment, the dendritic spine density in 4- to 6-month-old APP KO mice remained constant (Figs. 1C,D, $2 A$ ), with unchanged densities of persistent (Fig. 2C) and transient spines (Fig. 2D). At the same time, the survival fraction of dendritic spines in APP KO mice was similar to those in WT (Fig. $2 B)$. The observation that the pharmacological inhibition of $\gamma$-secretase activity, either by DAPT or LY450139, had no effect on dendritic spines in APP KO mice demonstrated that the GSIinduced decrease in dendritic spine density in WT mice is dependent on APP.

\section{Discussion}

Our results show two interesting findings. First, adult APP KO mice show a significantly increased spine density in the cerebral cortex, and second, $\gamma$-secretase inhibition leads to a reduction of dendritic spines in an APP-dependent manner.

The enhanced spine density in the cerebral cortex of APP KO mice supports previous in vitro findings showing that cultured hippocampal neurons from newborn mice as well as hippocampal sections from adult APP KO mice exhibit an increased number of synaptic endings (Priller et al., 2006). Detailed patch-clamp studies in autaptic hippocampal neurons further revealed that a lack of APP increases the number of synapses but does not affect the function of individual excitatory synapses either presynaptically or postsynaptically (Priller et al., 2006). The present study observed a close dose-response correlation between the expressed amount of APP and the number of dendritic spines in the cerebral cortex, since heterozygote APP litters show fewer spines than APP KO mice but more than WT mice. This result is in line with the previously proposed view that $\mathrm{A} \beta$ reduction is beneficial for excitatory synaptic transmission and dendritic spine density
(Kamenetz et al., 2003; Hsieh et al., 2006). Interestingly, an alternative way to reduce $A \beta$ levels via the pharmacological inhibition of $\gamma$-secretase activity in 4- to 6-month-old WT mice did not enhance dendritic spine density. In contrast, $\gamma$-secretase inhibition resulted in a persistent reduction of dendritic spine density in vivo. This effect was directly linked to the presence of APP, since the dendritic spine density did not decrease after $\gamma$-secretase inhibition in APP KO mice. However, whether this negative effect on dendritic spine density can also be observed in aged WT mice (Lanz et al., 2003) was not analyzed. Together, chronic GSI treatment over 4 consecutive days led to a loss of dendritic spines, whereas acute GSI treatment in previous studies was not found to alter spine density in organotypic hippocampal slices (Kamenetz et al., 2003) or cognition (Comery et al., 2005). We did not analyze whether the spine loss after chronic $\gamma$-secretase inhibition actually affects cognition. However, based on the results obtained from presenilin conditional KO mice (Saura et al., 2004), it is tempting to speculate that this may occur.

In addition to the advantageous reduction of $\mathrm{A} \beta$ after $\gamma$-secretase inhibition, other APP cleavage products seem to negatively modulate dendritic spine density in vivo. An involvement of the secreted N-terminal APP fragments $\operatorname{SAPP} \alpha$ and $\operatorname{sAPP} \beta$ is not very likely because they are largely unaffected by $\gamma$-secretase inhibition (Dovey et al., 2001). We rather suggest that the APPderived $\gamma$-secretase substrates C83 and/or C99, respectively, APP-CTFs that accumulate during $\gamma$-secretase inhibition, are toxic to dendritic spines in vivo. By these means, after $\gamma$-secretase inhibition, the synaptotoxic effect of APP-CTF accumulation might exceed the beneficial reduction of $\mathrm{A} \beta$, finally resulting in a decreased dendritic spine density in vivo. In this context, the toxic effect of $\gamma$-secretase inhibition on dendritic spine density in the adult mouse brain should make evident possible side effects of therapeutic approaches that lower $\mathrm{A} \beta$ but, on the other hand, increase the levels of C-terminal APP fragments.

\section{References}

Comery TA, Martone RL, Aschmies S, Atchison KP, Diamantidis G, Gong X, Zhou H, Kreft AF, Pangalos MN, Sonnenberg-Reines J, Jacobsen JS, Marquis KL (2005) Acute gamma-secretase inhibition improves contextual fear conditioning in the Tg2576 mouse model of Alzheimer's disease. J Neurosci 25:8898-8902.

Dovey HF, John V, Anderson JP, Chen LZ, de Saint Andrieu P, Fang LY, Freedman SB, Folmer B, Goldbach E, Holsztynska EJ, Hu KL, JohnsonWood KL, Kennedy SL, Kholodenko D, Knops JE, Latimer LH, Lee M, Liao Z, Lieberburg IM, Motter RN, et al. (2001) Functional gammasecretase inhibitors reduce beta-amyloid peptide levels in brain. J Neurochem 76:173-181.

Feng G, Mellor RH, Bernstein M, Keller-Peck C, Nguyen QT, Wallace M, Nerbonne JM, Lichtman JW, Sanes JR (2000) Imaging neuronal subsets in transgenic mice expressing multiple spectral variants of GFP. Neuron 28:41-51

Fleisher AS, Raman R, Siemers ER, Becerra L, Clark CM, Dean RA, Farlow MR, Galvin JE, Peskind ER, Quinn JF, Sherzai A, Sowell BB, Aisen PS, Thal LJ (2008) Phase 2 safety trial targeting amyloid beta production with a gamma-secretase inhibitor in Alzheimer disease. Arch Neurol 65:1031-1038.

Fuhrmann M, Mitteregger G, Kretzschmar H, Herms J (2007) Dendritic pathology in prion disease starts at the synaptic spine. J Neurosci 27:6224-6233.

Geling A, Steiner H, Willem M, Bally-Cuif L, Haass C (2002) A gammasecretase inhibitor blocks Notch signaling in vivo and causes a severe neurogenic phenotype in zebrafish. EMBO Rep 3:688-694.

Gitter BD, Czilli DL, Dieckman DK, Bender MH, Nissen JS (2004) Stereoselective inhibition of amyloid beta peptide secretion by LY450139, a novel functional gamma secretase inhibitor. Neurobiol Aging 25:571.

Grutzendler J, Kasthuri N, Gan WB (2002) Long-term dendritic spine stability in the adult cortex. Nature 420:812-816. 
Haass C (2004) Take five-BACE and the gamma-secretase quartet conduct Alzheimer's amyloid beta-peptide generation. EMBO J 23:483-488.

Holtmaat A, Wilbrecht L, Knott GW, Welker E, Svoboda K (2006) Experience-dependent and cell-type-specific spine growth in the neocortex. Nature 441:979-983.

Holtmaat AJ, Trachtenberg JT, Wilbrecht L, Shepherd GM, Zhang X, Knott GW, Svoboda K (2005) Transient and persistent dendritic spines in the neocortex in vivo. Neuron 45:279-291.

Hsieh H, Boehm J, Sato C, Iwatsubo T, Tomita T, Sisodia S, Malinow R (2006) AMPAR removal underlies Abeta-induced synaptic depression and dendritic spine loss. Neuron 52:831-843.

Kamenetz F, Tomita T, Hsieh H, Seabrook G, Borchelt D, Iwatsubo T, Sisodia S, Malinow R (2003) APP processing and synaptic function. Neuron 37:925-937.

Knott GW, Holtmaat A, Wilbrecht L, Welker E, Svoboda K (2006) Spine growth precedes synapse formation in the adult neocortex in vivo. Nat Neurosci 9:1117-1124.

Koh IY, Lindquist WB, Zito K, Nimchinsky EA, Svoboda K (2002) An image analysis algorithm for dendritic spines. Neural Comput 14:1283-1310.

Kopan R, Ilagan MX (2004) Gamma-secretase: proteasome of the membrane? Nat Rev Mol Cell Biol 5:499-504.

Lanz TA, Himes CS, Pallante G, Adams L, Yamazaki S, Amore B, Merchant KM (2003) The gamma-secretase inhibitor $N$-[N-(3,5-difluorophenacetyl)-Lalanyl]-S-phenylglycine t-butyl ester reduces A beta levels in vivo in plasma and CSF in young (plaque-free) and aged (plaque-bearing) $\operatorname{Tg} 2576$ mice. J Pharmacol Exp Ther 305:864-871.

Li ZW, Stark G, Götz J, Rülicke T, Gschwind M, Huber G, Müller U, Weissmann C (1996) Generation of mice with a 200-kb amyloid precursor protein gene deletion by Cre recombinase-mediated site-specific recombination in embryonic stem cells. Proc Natl Acad Sci U S A 93: $6158-6162$.

Majewska AK, Newton JR, Sur M (2006) Remodeling of synaptic structure in sensory cortical areas in vivo. J Neurosci 26:3021-3029.

Ness DK, Boggs LN, Hepburn DL, Gitter B, Long GG, May PC, Piroozi KS, Schafer KA, Yang Z (2004) Reduced amyloid burden, increased C-99 concentrations and evaluation of neuropathology in the brains of PDAPP mice given LY450139 dihydrate daily by gavage for 5 months. Neurobiol Aging 25 [Suppl 2]:238.

Priller C, Bauer T, Mitteregger G, Krebs B, Kretzschmar HA, Herms J (2006) Synapse formation and function is modulated by the amyloid precursor protein. J Neurosci 26:7212-7221.

Saura CA, Choi SY, Beglopoulos V, Malkani S, Zhang D, Shankaranarayana Rao BS, Chattarji S, Kelleher RJ 3rd, Kandel ER, Duff K, Kirkwood A, Shen J (2004) Loss of presenilin function causes impairments of memory and synaptic plasticity followed by age-dependent neurodegeneration. Neuron 42:23-36.

Selkoe DJ (1994) Alzheimers-Disease-a central role for amyloid. J Neuropathol Exp Neurol 53:438-447.

Selkoe DJ (2002) Alzheimer's disease is a synaptic failure. Science 298: 789-791.

Selkoe DJ, Wolfe MS (2007) Presenilin: running with scissors in the membrane. Cell 131:215-221.

Spires TL, Meyer-Luehmann M, Stern EA, McLean PJ, Skoch J, Nguyen PT, Bacskai BJ, Hyman BT (2005) Dendritic spine abnormalities in amyloid precursor protein transgenic mice demonstrated by gene transfer and intravital multiphoton microscopy. J Neurosci 25:7278-7287.

Terry RD, Masliah E, Salmon DP, Butters N, DeTeresa R, Hill R, Hansen LA, Katzman R (1991) Physical basis of cognitive alterations in Alzheimer's disease: synapse loss is the major correlate of cognitive impairment. Ann Neurol 30:572-580.

Trachtenberg JT, Chen BE, Knott GW, Feng G, Sanes JR, Welker E, Svoboda $\mathrm{K}$ (2002) Long-term in vivo imaging of experience-dependent synaptic plasticity in adult cortex. Nature 420:788-794.

Tsai J, Grutzendler J, Duff K, Gan WB (2004) Fibrillar amyloid deposition leads to local synaptic abnormalities and breakage of neuronal branches. Nat Neurosci 7:1181-1183.

Zuo Y, Yang G, Kwon E, Gan WB (2005) Long-term sensory deprivation prevents dendritic spine loss in primary somatosensory cortex. Nature 436:261-265. 